\title{
LA ESCENA POLÍTICA DE LOS MOVIMIENTOS DE LAS MUJERES EN COSTA RICA DURANTE LOS ÚLTIMOS TREINTA AÑOS
}

\section{POLITY MOVEMENTS OF WOMEN SCENE IN COSTA RICA DURING THE LAST THIRTY YEARS}

\author{
Nancy Piedra Guillén ${ }^{1}$ \\ npiedraguillen@yahoo.com
}

Fecha de recepción: 29 agosto 2014 - Fecha de aceptación: 5 mayo 2015

\begin{abstract}
Resumen
Este ensayo intenta reflexionar sobre los movimientos sociales de mujeres y feministas en Costa Rica en los últimos 30 años. Se analizan sus cambios internos, su impacto en la escena política, sus aciertos y sus limitaciones. El ensayo describe el origen y el desarrollo de algunos grupos surgidos en las décadas de 1980 y 1990 y los contrasta con los grupos de la última década del presente siglo. Se concluye que la presencia de los movimientos de mujeres y/o feministas es de particular importancia para hacer avanzar la lucha de las mujeres en general, en favor de la equidad y la igualdad de condiciones entre los sexos/ géneros. La reflexión crítica sobre la vida de las mujeres, las diferencias, las discriminaciones y los vacíos de las políticas públicas requieren claridad política y conciencia sobre los factores determinantes de la desigualdad. Este aspecto tiende a diluirse en los discursos de instituciones públicas, partidos políticos, organismos no gubernamentales y organismos internacionales. Tales discursos tienen propuestas limitadas, acotadas a aspectos no controversiales, que por lo general promueven roles tradicionales de género. Por el contrario, las feministas defienden rupturas socioculturales y económicas que favorecerían cambios transcendentales para las mujeres en su conjunto.

Palabras claves: participación política de las mujeres- movimientos sociales de mujeres - movimientos feministas - colectivos LGBTI - necesidades estratégicas de género.
\end{abstract}

\begin{abstract}
This essay addresses the women and feminist social movements in Costa Rica during the last thirty years and analyses their internal changes, their impact in Costa Rican polity, and their successes and shortcomings. The essay presents the origins and developments of some of the groups arisen in the 1980s and 1990s and compares them with similar groups born in the last ten years. The essay concludes that women and feminist movements are cardinal for the advance of all women's fights for equity and equality in gender and sexual conditions. Critical reflection on women's life, differences and discriminations, and on the gaps in public policies for women requires an adequate political understanding and a sharp consciousness about the sources of gender inequality. This vital question disappears very often in the discourses of public institutions, political parties, ONGs and international organizations. Instead, these discourses put forward limited solutions for women's demands; they remain in the frame of non-controversial matters and promote traditional gender roles. On the contrary, feminist movements are in favor of socio-cultural breakthroughs that would bring fundamental changes for all women.

Key words: Political Participation of Women-Women's Social Movements - Feminist Movement Collective LGBTI - Strategic Gender Needs.
\end{abstract}

1 Docente de la Escuela de Sociología, de la Universidad de Costa Rica. 


\section{Introducción}

Este artículo trata sobre un tema poco abordado en los análisis académicos en años recientes: los movimientos sociales de las mujeres y su presencia en la escena política del país. Retomamos el tema de la presencia política de las mujeres con el fin de reflexionar sobre aspectos particulares relacionados con la naturaleza de los movimientos de mujeres en las últimas tres décadas, se apela asincrónicamente a hechos y procesos organizativos para reflexionar sobre la temática de interés. Momento de particular pertinencia por demandas y procesos organizativos recientes que nos permiten reflexionar a su vez en hechos del pasado y, preguntarnos: ¿qué ha pasado durante las dos últimas décadas del siglo pasado y lo que va del presente siglo con los movimientos sociales de las mujeres? ¿Cuánto se distancian y diferencian los procesos organizativos de las mujeres de los ochenta y noventa del siglo pasado con los actuales? ¿Se han dado cambios sustanciales en la escena política?

Aunque el artículo no forma parte de una investigación de carácter empírico, para realizarlo se hizo una revisión bibliográfica sistemática sobre el tema, se buscó información sobre las organizaciones en internet y posteriormente se contactó a las mismas para corroborar la información y actualizar los datos que se encontraron en las referencias. También se hicieron varias entrevistas a dirigentes de distintos partidos políticos, en el actual escrito solo se retoma una de ellas por la pertinencia con el tema tratado.

Se parte de dos tesis principales para sustentar la reflexión desarrollada: 1. En la actualidad, organismos internacionales, organismos gubernamentales y no gubernamentales y organizaciones de mujeres se han apropiado de un discurso que ha sido generado por los movimientos de mujeres y feministas. Sin embargo, las prácticas y programas que desarrollan dichas instituciones no son lo suficientemente críticos y novedosos para promover la igualdad y la equidad de género. Para generar procesos de ruptura promotores de cambios sustanciales en el quehacer político y cultural de las mujeres, es necesario tener conciencia y actitud crítica ante la realidad. Ambos son actos racionalizados que se proyectan políticamente y deben impactar la sociedad. Por el contrario, los programas de los partidos y otras organizaciones no específicamente de mujeres han estado restringidos a ciertas áreas, no se logran rupturas y por ello algunos problemas tienden a reproducirse y/o agudizarse. 2. Los movimientos se retroalimenta de acciones promovidas por las mujeres en tanto sujetas individuales y colectivas, prácticas que permiten generar procesos de inflexión sociales y/o individuales transformadoras. Si los nuevos valores no se fortalecen en la cotidianidad y/o permean las políticas y esquemas culturales, se tenderán a reproducir las estructuras sociales tradicionales que impiden los cambios.

Durante las décadas de los ochenta y noventa del siglo pasado, los movimientos sociales y la participación política de las mujeres marcó un cambio en la escena política en América Latina, y de forma particular en nuestro país. A diferencia del movimiento de mujeres en Europa y Norte América, el de América Latina tuvo en dichas décadas una contundente presencia en espacios públicos, sus demandas se ampliaron y respondieron tanto a las llamadas "necesidades prácticas de género", como a las "necesidades estratégicas de género". ${ }^{2}$ Actividad política que no se observaba con tal fuerza desde el movimiento sufragista de los años 40 del siglo pasado (Barahona: 1987 y Piedra, 1998).

2 Se ha decidido retomar las categorías de análisis de Moser (1992) porque desde nuestra perspectiva siguen siendo válidas para comprender los alcances y limitaciones del quehacer político de las mujeres en la actualidad. Las necesidades prácticas de género, en la medida en que responden a carencias que se asocian a la condición de mujeres como ser madres, cuidadoras de las familias, y principales responsables del trabajo doméstico, generaron en la década de los años ochenta del siglo XX valiosos proyectos como "Comedores populares" y "El vaso de leche" en el sur de América Latina, experiencias que se desarrollaron para enfrentar los efectos de la crisis económica de esos años (Vargas, 1992, 1997 y Piedra, 1998). Por su parte, las "necesidades estratégicas de género" buscan cambios en la posición, situación y condición de vida de las mujeres. La idea es posicionarlas en espacios de poder de forma trascendente para romper con los roles tradicionales de género que han impedido que se produzcan cambios de fondo en las relaciones tradicionales con respecto a los hombres, sea en el acceso a estructuras de poder político, económico, social, laboral, cultural, simbólicos, así como cambios en las relaciones al interior de las familias (Moser, 1992, 1995 y Young, 1993). 
En recientes investigaciones se constata (Piedra, 2011) las transformaciones que se han dado en las sociedades occidentales a lo largo de las pasadas décadas, pero estas se han generado de forma paulatina. Persisten prácticas tradicionales, como la inequitativa distribución del trabajo doméstico, que son factores que afectan a la mayoría de las mujeres, puesto que dicha desigualdad repercute en el desarrollo y desempeño de ellas en otros ámbitos de la vida social, profesional, política, entre otras. De ahí que, reivindicaciones como las "cuotas de participación política", la lucha contra la violencia hacia las mujeres y el derecho a decidir sobre sus vidas y cuerpos siguen siendo válidas, medulares y polémicas, porque centran la lucha de las mujeres en aspectos fundamentales que tienen que ver con las desigualdades de género. Son esas problemáticas las que han impulsado a cientos de mujeres a organizarse y manifestarse de forma individual y colectiva.

El texto ha sido organizado por secciones, con el fin de tratar distintos aspectos que tienen que ver con las preocupaciones presentadas anteriormente. El primer y el segundo apartado introducen el tema del movimiento de mujeres y de los movimientos sociales en general a nivel teórico para, posteriormente, dedicar el tercer apartado al análisis de la participación de las mujeres en la escena política durante las dos últimas décadas del siglo XX. En el cuarto, se identifica y analiza la situación de los movimientos y otras expresiones organizativas de las mujeres del siglo XXI y en el quinto subapartado se hace referencia a los colectivos lésbico-gay-bisexual-transgénero-intersexo (LGBTI). Posteriormente, nos preguntamos si en la actualidad los movimientos feministas de mujeres y de la diversidad sexual deben asumir los principios del poscolonialismo, lo cual los distanciaría en alguna medida de los movimientos occidentales, rasgo que ha estado presente y ha sido importante en los movimientos sociales de las mujeres.

\section{Aspectos conceptuales y escena política de los movimientos sociales contemporáneos}

En los años 70 del siglo XX, en Europa y Estados Unidos, a diversos movimientos de protesta se les denominó "nuevos" movimientos sociales, porque desbordaron la lógica analítica con la cual habían sido estudiados hasta entonces los movimientos, en particular la relación explicativa entre privaciones materiales y sociales.

Se inicia así, una distinción entre los denominados viejos y nuevos movimientos sociales. Los primeros son asociados con preocupaciones concernientes principalmente al ámbito de lo económico (crecimiento económico, distribución y consumo), en tanto que, los "nuevos movimientos sociales" fueron "asociados con los territorios, los espacios de acción, la vida en el mundo del cuerpo (salud, identidad sexual) la herencia e identidad cultural, étnica, nacional y lingüística; las condiciones físicas de vida y la supervivencia de la raza humana en general" (Offe, 1985:ح929).

Los "nuevos movimientos sociales" o los "movimientos contemporáneos", tal como les llama Offe (1985), se caracterizan porque la clase social a la que pertenecen las personas y los códigos políticos establecidos (izquierda/derecha) ya no serán determinantes como colectivo; toma mayor importancia su identidad con respecto al género, sexo, edad, localidad, aspectos culturales y visión de vida. La dinámica organizativa tiende a fluir a través de redes de relaciones informales. Estas redes van desde el pequeño grupo vecinal, de mujeres, ecologista, u otros, hasta las redes nacionales e internacionales que tienden ahora a globalizarse, puesto que las tecnologías de la información y comunicación lo facilitan (Castells, 1998, 2001).

Quienes exaltan los conceptos de los agentes sociales como sujetos de transformación atribuyen un mayor peso a lo que piensan y sienten dichos actores sociales, a las modificaciones en sus valores y a su disposición a la acción colectiva; plantean y defienden sus intereses en la esfera pública (Offe, 1985). Por su parte, Touraine (1995) subraya la importancia de diferenciar aquellos procesos de protesta colectiva o lucha que por la especificidad de sus demandas no representan un movimiento social, acepción de la que partimos en el presente texto. Es decir, un movimiento social es aquella acción conflictiva mediante la cual se transforman las orientaciones culturales que están definidas por normas culturales 
generales y por relaciones de dominación social, pero las luchas no están dirigidas solamente contra un orden imperante particular, sino que se actúa en nombre de valores considerados esenciales por el conjunto de la sociedad, aunque no todos compartan una reivindicación en específico.

En ese caso, los movimientos feministas y de mujeres, los colectivos lésbico-gay-bisexual-transgénero-intersexo LGBTI y los movimientos ambientalistas, entre otros, se caracterizan porque presentan reivindicaciones que atañen a "patrones culturales a través de los cuales una colectividad constituye de manera normativa sus relaciones con el entorno" (Touraine, 1995: 255). Así que los movimientos sociales se manifiestan en la oposición de intereses y de las luchas que se desarrollan en un proceso de confrontación, negociación y diálogo.

Los sujetos sociales tienen grados de organización y estabilidad variable (Ciriza, 2007) característica que los identifica y diferencia de otras expresiones políticas, como los partidos políticos o los sindicatos, que tienden a construir estructuras organizativas que les permite mantenerse de forma más estable y duradera.

En el caso de América Latina, los movimientos contemporáneos se empiezan a gestar a finales de la década del 70 y cobran fuerza y un mayor protagonismo en la década de los años 80 del siglo pasado y coinciden con los movimientos europeos cuyas demandas giran en torno a temas y problemas de interés como el medio ambiente, cambio climático, y feministas, entre otros. En este sentido, los nuevos enfoques teóricos sobre los movimientos sociales en América Latina reconocen la participación protagónica de los sectores populares urbanos y rurales en ellos, así como nuevas formas de organización y movilización política mediante las cuales se intenta transformar situaciones estructurales que afectan a diferentes sectores sociales.

\section{Las mujeres en movimiento}

Se sabe que las mujeres no constituyen un grupo social en sí, sino que se trata de una categoría transversal a las clases, a los grupos culturales, a las comunidades, a las naciones, a los territorios. "De ahí la ambivalencia del espejo. Instancia de reconocimiento imaginario, atravesada por una línea de ficción, la ambivalencia especular que puede ser parcialmente simbolizada" (Ciriza, 2007: 29). Se está ante un movimiento constituido por identidades diversas. Por medio de su práctica ha logrado integrar a mujeres de las más variadas expresiones, lo que le da una riqueza particular. La heterogeneidad es tan palpable como sus demandas, de ahí que se prefiera referirse al mismo en forma plural y no singular.

Para comprender la diversidad, autoras como Vargas (1992) clasificaron los movimientos sociales de mujeres en vertientes según el tipo de acciones y demandas. Estas categorías nos permiten comprender las distintas expresiones organizativas. Al respecto, Vargas menciona tres vertientes: las feministas, las de las mujeres que buscan satisfacer necesidades y demandas que surgen de sus roles tradicionales, y las de mujeres con presencia en espacios públicos tradicionales como partidos políticos, sindicatos y asociaciones.

En su configuración, los movimientos de mujeres y feministas latinoamericanos no han mostrado un proceso homogéneo, sino la pluralidad de procesos que evidencian la diversidad de las realidades de las mujeres. No existe en los movimientos de mujeres una única forma de percibir y expresar la subordinación de género y sexo, por ello cada expresión organizativa o sector que compone al movimiento se ha desarrollado y evolucionado de acuerdo con las situaciones sociales específicas que les ha tocado vivir. Desde nuestra perspectiva, podemos decir que las mujeres crean movimientos y procesos colectivos a partir de sus historias, sus experiencias de vida y de sus referencias simbólicas y prácticas (Piedra, 1998 y Vargas, 1992). En dicho proceso van cambiando ellas mismas, su visión de mundo, sus conductas, expectativas, sueños, capacidad de acción e identidades, experiencia que quizás también sea similar en otros movimientos sociales.

Los móviles de la organización pueden tener distintos puntos de partida -alimentación, organización política, asuntos de género- desde el cual se cuestiona la subordinación de género y se construye a la vez él o los movimientos. Hay procesos de transformación social que impactan el mundo de lo político y las 
políticas públicas, pero también se han generado procesos de transformación personal, que impactan la vida íntima, sea en la relación de pareja, la familia, el espacio social y público como el trabajo, el partido, la organización, la escuela, el centro de salud. Llama la atención que, cada vez más, a diferencia de lo que ocurría en la década de los 80 s, mujeres de distintos sectores económicos que no pertenecen a los movimientos sociales, logran reconocer sus derechos. Aunque el reconocimiento de los derechos no implica una identificación con organizaciones de mujeres y menos aún, con feministas (Piedra, 2011 y 2013).

El feminismo es una vertiente o sector de los movimientos de mujeres que trabaja a conciencia para resquebrajar y transformar el sexismo y las relaciones de poder inequitativas que viven las mujeres por su condición de género y sexo, en los diferentes ámbitos de la sociedad. Así que, retomando a Vargas (1992) y Moser (1992), podemos decir que las expresiones organizativas de las feministas son procesos colectivos de mujeres y grupos afines que se preocupan por plantear las necesidades estratégicas de género, las cuales se formulan a partir del análisis de la subordinación de las mujeres en relación a los hombres.

Según Vargas, la segunda vertiente dentro del movimiento social de mujeres está compuesta por mujeres que buscan satisfacer necesidades y demandas que surgen de sus roles tradicionales. Al organizarse y luchar por alcanzar ese fin, estas dan un contenido más público a esos roles, logrando hacer de sus actividades individuales esfuerzos colectivos para promover un mundo más justo. Pero, en un mundo globalizado, la escena de lo político se torna cada vez más compleja a tal punto que conceptos claves en la lucha de las mujeres, como el de justicia, son necesarios revisarlos, no solo en lo relativo a "los contenidos de lo aún adecuado en materia de justicia en las comunidades políticas, sino también en relación con quienes constituyen actualmente la comunidad y cuál es la comunidad relevante a la que el concepto debería referirse en su extensión" (Fraser en Rigat, 2014: 134). Esto hace que la escena política en la que actúan las mujeres sea sumamente compleja.

La tercera vertiente surge de los espacios públicos tradicionales como partidos políticos, sindicatos, o asociaciones, entre otros. Son principalmente mujeres y/o feministas con compromiso con respecto a su género, intentan abrir nuevos espacios para las mujeres, para lograr una mayor participación en la toma de decisiones en el nivel local y nacional. Luchan por un reconocimiento formal y más público de los derechos femeninos, nos señala Ligia Fallas (entrevista, PFA), con la limitación, de atender más las necesidades prácticas de género y no las estratégicas.

Lo interesante es observar cómo, planteamientos como los de Vargas (1992) y Moser (1992), formulados en los años noventa, siguen teniendo relevancia y pertinencia para comprender las limitaciones de las acciones contemporáneas, a pesar de la diferencia temporal y las experiencias organizativas de las mujeres, aportando explicaciones para entender las limitaciones de los procesos actuales. Por lo tanto, a pesar de las diferencias que existen, lo que se sostiene en este artículo es que los movimientos de las mujeres han sido reconocidos porque han logrado impactar a las sociedades contemporáneas y en tanto que dinámica y expresión de diversidad se mantienen en la palestra política, mostrando capacidad de cambio y adaptación para continuar con su cometido principal: luchar por la defensa de los derechos de las mujeres, en pro de la igualdad y la equidad en lo político, económico y cultural, y más recientemente cada vez más se manifiestan a favor de la liberación de sus cuerpos, la sexualidad y el respeto a la diversidad. Sin duda, la forma en la que se expresan actualmente los movimientos sociales de mujeres se ha transformado, por lo que nos preguntamos si habrá acumulación en las propuestas políticas y organizativas. ¿Hay rupturas estructurales o se ha institucionalizado su lucha?, y por ello los movimientos han aminorado su presencia política.

\section{La escena política de las mujeres}

Sin duda, hace varios años, existió en el país la necesidad de reflexionar sobre las prácticas políticas de las mujeres y con ello analizar las posibilidades de cambio en ellas y la sociedad, ello dio origen a varios textos que analizaban la conformación de los movimientos sociales y participación política de las mujeres (Flores, 1993; Lara, 1994; Moreno, 1995; Camacho, Lara y Serrano, 1996; Cordero, 1996; 
Sagot, 1997; Piedra, 1998 y 1999 e Hidalgo, 2004). En este espacio interesa, a manera de síntesis, destacar algunas acciones que contribuyeron al desarrollo y consolidación de los movimientos de mujeres en las dos últimas décadas del siglo XX; para posteriormente identificar las y los protagonistas de los movimientos sociales de mujeres y/o feministas del presente.

A continuación destacamos momentos claves, organizaciones y espacios que a nuestro juicio fueron centrales en las dos últimas décadas del siglo XX y que llegan incluso hasta el presente:

1. Desde finales de la década de los años 70 , los movimientos sociales de mujeres/feministas se caracteriza por un dinamismo que va creciendo durante los años 80 y 90 . Se fundan organizaciones que fueron referencia para mujeres de escasos recursos económicos de las zonas urbanas y rurales del país, entre las más conocidas mencionemos a: grupos feministas como Alianza de Mujeres Costarricenses (1948), Centro Feminista de Información y Acción (Cefemina) (1979), Colectivo de Mujeres Pancha Carrasco (1987), Asociación Carmen Lyra; grupos lésbicos como Ventana (1979), La Colectiva Lésbica (1986), Las Entendidas (1986), Las Humanas (1990), Gaypoa (1990), Grupo 5 de abril (1995), Las Diosas de Luna y Miel (1995), Triángulo Rosa (1995). Así como, instancias de coordinación como el Comité Latinoamericano de Defensa de los Derechos de la Mujer CladenCosta Rica (1988), Centro de Investigación y Promoción para América Central Cipac (1999) (Piedra, 1998 y Torres, 2012).

2. En los años 80 se acude a la constitución de equipos de trabajo de mujeres líderes y activistas al interior de las organizaciones gremiales mixtas como los sindicatos y las cooperativas. Se organizaron grupos comunales tanto urbanos como rurales alrededor principalmente de demandas prácticas de género. Además, "proliferan los grupos productivos de mujeres organizados por la vía cooperativista, asociativa o micro empresarial, estimándose la existencia de unos 480 grupos de este tipo a mediados de los 80s". (Quesada y Camacho, s.f.:5).

3. Se gestan espacios de coordinación que fueron muy importantes y propiciaron la puesta en marcha de ejes de lucha en común, como: La Colectiva 25 de noviembre (1989), la Colectiva Feminista Nacional (1991), Consejo de Mujeres Indígenas, la Asociación de Mujeres Afrocaribeñas, la Asociación Nacional de Mujeres con Discapacidad, la Red de Mujeres en Acción, la Asociación Nacional de Madres Comunitarias (1990). La Red de Mujeres Ecuménicas y Pastoras (1988) que da origen a la Red de Mujeres Trabajando con Mujeres (1989), la Coordinadora de Mujeres Campesinas CMC e iniciativas de jóvenes como el Grupo de Mujeres Antítesis. Finalmente, destaquemos el espacio de coordinación llamado La Agenda Política de las Mujeres (1997), iniciativa de coordinación que se propuso impulsar temas y demandas de las mujeres para ser negociadas con partidos políticos y/o instituciones (Piedra, 1998, 1999).

4. Por otra parte, se dieron espacios de Encuentro de particular importancia como: I Encuentro Centroamericano de Mujeres: "Una Nueva Mujer un Nuevo Poder" (marzo de 1993), "VI Encuentro Feminista Latinoamericano y del Caribe" realizado en El Salvador (noviembre de 1993), siendo el mismo el primer Encuentro Feminista, realizado en la región y organizado por centroamericanas.

5. A su vez, el escenario de las organizaciones y movimientos tuvo como puntos focales de interés las cinco Conferencias Mundiales de las Naciones Unidas dirigidas a las mujeres, lo que motivó la creación de espacios de coordinación y se elaboraron documentos que fueron presentados en ellas.

Las iniciativas organizativas y de coordinación aquí mencionadas no dan cuenta de todas las acciones que se llevaron a cabo, pero son suficientes para considerar el carácter dinámico que caracterizó los movimientos del período analizado. Pensamos que, si algo caracterizó a las diversas expresiones organizativas de este período fue la necesidad y la capacidad para forjar espacios de encuentro que permitieron el diálogo, el debate y el acercamiento que se observó en los distintos encuentros nacionales, centroamericanos y latinoamericanos. Espacios de intercambio que hicieron posible el establecimiento de acuerdos mínimos y apoyos estratégicos de unas organizaciones con respecto a otras. 
Actualmente son múltiples las expresiones organizativas de las mujeres, que van de las ya legendarias y mencionadas; a las conformadas en medio del auge organizativo de los años noventa como Mujeres por un Desarrollo Alternativo Somudal, Red de Mujeres en Acción, Fundación Genero y Sociedad GESO, entre otras. Ello sin mencionar grupos y comités barriales que existen en comunidades urbanas y rurales del país. Sin embargo, la mayoría de las organizaciones han ido delimitando su trabajo a aspectos que responden más a las necesidades prácticas de género. Actualmente, se privilegia trabajar en aspectos relacionados con la autonomía económica y se deja de lado procesos de reflexión, de empoderamiento personal y político, al menos así lo reflejan sus objetivos y la poca presencia política. Las instituciones por su parte, no tienden a promover la conciencia de las mujeres en tanto acto racionalizador e impulsor de acciones estratégicas que contribuyan a romper con las barreras sociales y culturales, basadas en las diferencias de género y sexo. Aspecto que por lo demás, no les corresponde.

Por tanto observamos como la presencia política de las mujeres se va aminorando en los primeros años del presente siglo. Más no por ello, los movimientos estuvieron totalmente ausentes, lo que ha sucedió es que el escenario ha cambiado y con ello las temáticas, los intereses y las demandas. Es un período en el que se prioriza la gestión de políticas públicas y programas de desarrollo en busca del mejoramiento en la calidad de vida y acceso a oportunidades para las mujeres. Mientras que temas, como las cuotas de participación y la representación paritaria, toman un lugar preeminente.

Así, mientras un grupo importante de feministas líderes del país fueron llamadas o ya formaban parte de instituciones que generaban propuestas, los grupos y expresiones organizativas de mujeres y feministas se empieza a debilitar y/o a transformar. Decenas de líderes se incorporan a trabajar instituciones como el Instituto de la Mujer, IMAS, Ilanud u organismos internacionales. Se observa así un proceso de institucionalización que termina colapsando el proceso de efervescencia política y de activismo social que se vivió en las décadas anteriores.

\section{Otros momentos, otros protagonismos, la escena política en la actualidad}

Llama la atención, cómo en el pasado, grupos lésbicos tuvieron un papel relevante en el desarrollo de los movimientos de mujeres y feministas del país, al igual como en la actualidad grupos como: Las Humanas, Las Entendidas, El Reguero y Tertulia entre Mujeres, aunque no de forma exclusiva, han tenido un papel importante en la gestión de los colectivos de la diversidad LGBTI (Serrano, 2002). Además afirma Torres (2012) que los grupos gay tienden a tener una mayor presencia en la escena política y proclaman una visibilidad pública como mecanismo para hacer avanzar sus reivindicaciones; tal es el caso del Movimiento Diversidad (2004), el grupo de Lucha contra el SIDA para personas transgénero (2004), grupo de Jóvenes Prisma, la Coordinadora el Beso Diverso, entre otros. ${ }^{3}$ Los colectivos LGBTI se han caracterizado por la naturaleza de sus demandas, las cuales giran en torno a temas y problemas sumamente polémicos, empezando por cuestionar las pautas homofóbicas y heteronormativas del país, además de otros tópicos relacionados con los derechos humanos.

Por ejemplo, en Costa Rica el aborto es legal solo si se demuestra que la vida o la salud de la mujer embarazada están en riesgo. No existen protocolos de atención y menos aún normas administrativas para que se pueda llevar a cabo una interrupción sin llamar la atención de las autoridades médicas sobre la naturaleza de este. Fueron los colectivos LGBTI que en el 2007 retoman y promueven la discusión sobre las características del aborto terapéutico con el fin de ampliar el restringido criterio que ha imperado en la práctica. Se promueve el debate sobre la legalización voluntaria de la interrupción del embarazo: el derecho al aborto.

Igualmente son colectivos LGBTI los que han promovido la reivindicación del matrimonio entre personas del mismo sexo, plantearon el "Proyecto de Ley de Sociedades de Convivencia",

3 Se recomienda consultar el texto de Torres (2012) que da cuenta de las luchas lésbicas en el país. 
propuesta que fue rechazada por la Comisión de Derechos Humanos en la Asamblea Legislativa (6/06/2012). La reacción fue casi que inmediata de parte de grupos feministas, de mujeres, ecologistas y otros, liderados por los colectivos que representan al movimiento LGBTI, proceso que culminó con la marcha de "Los invisibles". El gobierno, ante la presión social, tuvo que manifestarse y se comprometió a mandar a sesiones extraordinarias el proyecto de "Ley de Sociedades de Convivencia" a la Asamblea Legislativa. (http://www.asamblea.go.cr/Centro_de_informacion/Sala_de_Prensa/Documents/ Exp.18481.pdf).

Posteriormente, el 7 de marzo del 2013, el Movimiento Diversidad, junto a la Defensoría de los Habitantes, la Iglesia Luterana, grupos de mujeres y personas civiles realizaron "La Marcha de los Incurables" se manifestaron en contra de la posición homofóbica del Gobierno de turno (administración Chinchilla 2010-2014), de algunas iglesias y el V Congreso Centroamericano de Bioética.

Observemos que las demandas del colectivo LGBT refieren a peticiones antiguas que siguen siendo válidas, problemas no resueltos en una sociedad que se debate entre posiciones progresistas y otras muy conservadoras y fundamentalistas, se sigue negando la realidad puesto que siguen ausentes en las normativas y en la legalidad de la sociedad costarricense. Otro aspecto a destacar es que el colectivo LGBTI cuando se manifiesta públicamente lo hace acompañado y apoyado por otros grupos de la sociedad civil y de movimientos progresistas como el ecologista, las feministas, algunas organizaciones de mujeres, religiosas. Incluyendo partidos políticos como el Frente Amplio PFA y Acción Ciudadana PAC (que hoy conforma el gobierno 2014-2018), los cuales se destacan por sus propuestas a favor del feminismo, las mujeres, los movimientos ambientales y en pro del respeto a las diversidades sexuales (entrevista a diputada Ligia Fallas).

Por otra parte, una diferencia que notamos es el protagonismo que tenían los movimientos feministas en los procesos de formación y capacitación de organizaciones de mujeres urbanas y rurales. Mientras que, a inicios del siglo XXI, dicha labor empieza a ser retomada por instituciones gubernamentales como el Instituto de la Mujer, INAMU, el Instituto Mixto de Ayuda Social, IMAS y otras instancias que desde su labor pública y normativa deben incorporar el enfoque de género y considerar a las mujeres como sujetas sociales de sus políticas.

Se sostiene entonces que este período culmina con la elaboración de la Política Nacional para la Igualdad y Equidad de Género 2007-2017 (INAMU, 2007). Dicha acción representa un paso adelante en la atención de las necesidades de las mujeres en general, pero ha contribuido también a despolitizar la actividad organizativa de ellas. El trabajo se orienta a fortalecer actividades productivas; que, tal como se señaló, responden a las "necesidades prácticas" de las mujeres de escasos recursos económicos. Mientras que las demandas de la violencia en contra de las mujeres queda en manos del INAMU como ente responsable de velar por la aplicación de la Ley en "Contra de la Violencia Intrafamiliar". Por ejemplo, el Día de la No Violencia contra las Mujeres es ahora organizado por esta entidad, siendo que en años anteriores lo hacían los movimientos de mujeres y la instancia de coordinación que se había creado para ese fin. La formación-capacitación que se dirige a las mujeres se centra en el estudio de sus derechos, enfatizándose la parte normativa de estos y aminorando la parte política donde subyacen. 
Manifestación en el Día de la No Violencia contra las Mujeres 25 de noviembre 1990

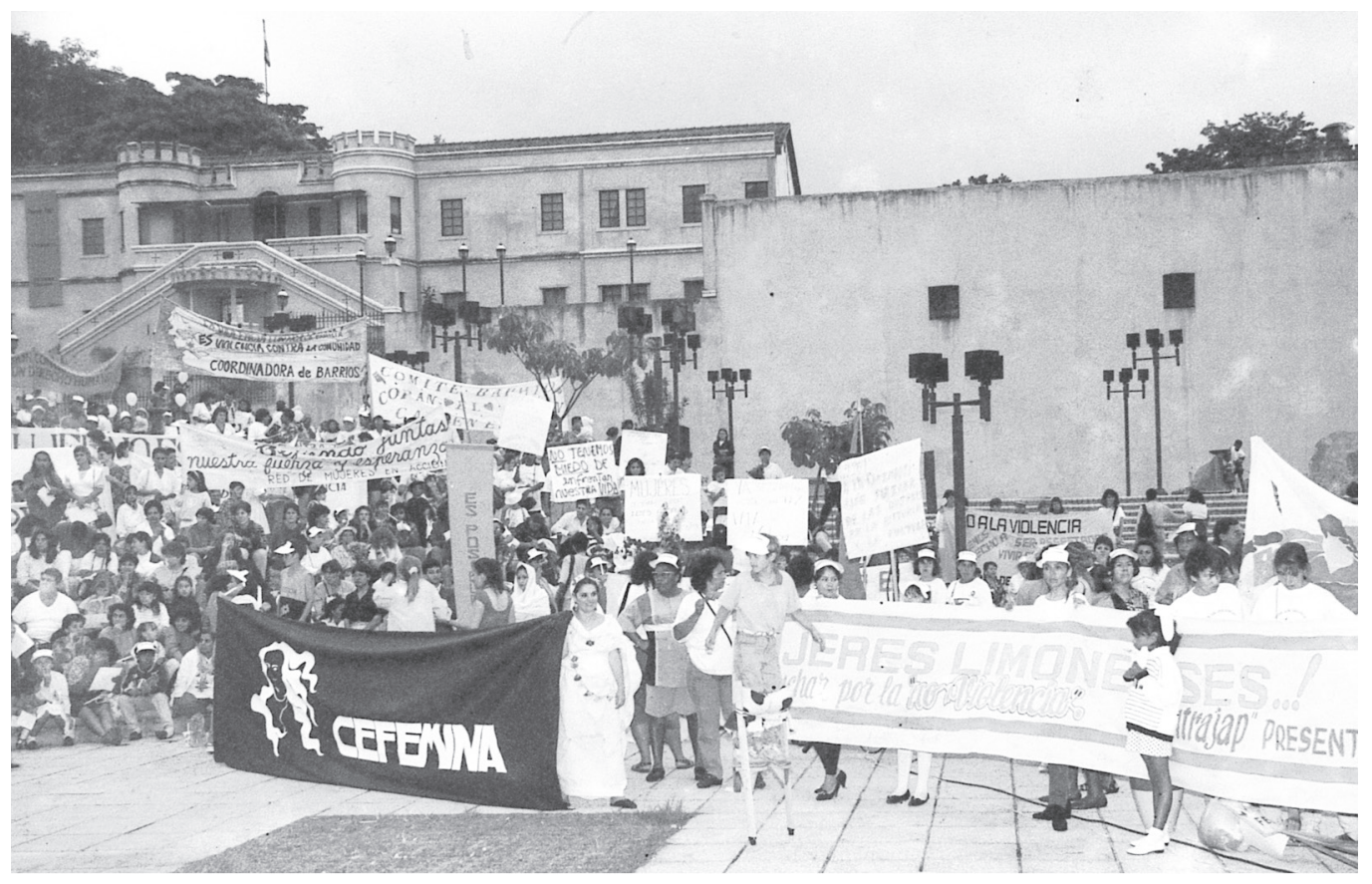

Fuente: Nancy Piedra Guillén, colección personal, 1990.

Por una parte, los logros y reivindicaciones de los movimientos del pasado han cedido a la institucionalización. Debilitando la base social del movimiento, sus demandas y autonomía; más aún los procesos de coordinación a nivel nacional y regional. Por otra parte, se avanza hacia la delimitación de otras acciones y temáticas que cobran importancia a nivel internacional y, posteriormente, nacional, como son las cuotas de participación, las políticas afirmativas y la presencia de estas en espacios partidistas y sindicales, entre otros.

De esta forma, en la primera década del siglo XXI mientras instancias gubernamentales subsumen las reivindicaciones de género de las mujeres, otras expresiones organizativas empiezan a resurgir o a gestarse como nuevas formas de replantearse la defensa de los derechos de las mujeres y las diversidades sexuales. Al observar la presencia de las nuevas organizaciones y movimientos, consideramos que en las dos décadas finales del siglo XX, los movimientos de mujeres tuvieron como protagonistas a mujeres de sectores urbanos y rurales de corte popular, que junto con distintas expresiones del feminismo generaron una base de apoyo a las reivindicaciones de género. Mientras que, en la segunda década del siglo XXI, las instancias protagonistas son los colectivos LGBTI conformados por personas que cuestionan la visión dicotómica que ha imperado en el análisis teórico y demandas en los movimientos feministas y de mujeres a nivel mundial. Los colectivos de la diversidad sexual señalan que la mayoría de las organizaciones feministas y de mujeres han centrado sus propuestas en el análisis de las diferencias entre hombres y mujeres y el posicionamiento privilegiado que históricamente han tenido los hombres; y dejan de lado, otros grupos sociales que no se identifican ni con los hombres, ni con las mujeres. 
Desde las estructuras de poder se ha permitido que los hombres mantengan privilegios propios de género fundados en el sistema patriarcal, que aunque se ha modificado a lo largo de la historia, se mantienen a pesar del generalizado cuestionamiento que se hace de este.

\section{¿Los feminismos ahora deben ser poscoloniales?}

Finalmente, cabe la pregunta: ¿los feminismos ahora deben ser poscoloniales?, ¿es esta una perspectiva de análisis más amplia e inclusiva? Sin duda es tema que merece un espacio propio de reflexión, pero que no se debe dejar de lado por la actualidad, importancia y auge que tiene en América Latina. Nos surgen algunas dudas, dado que no necesariamente el poscolonialismo nos lleva a comprender o entender todas las diferencias, aunque nos permite comprender las particularidades de muchas mujeres que hasta hace pocos años se han considerado personas sin voz propia y, más aún, sin poder. Por ello, cada vez han ganado más terreno las posturas de feministas que desde una lectura crítica al feminismo hegemónico - de matriz occidental- se plantean la necesidad de ampliar las agendas para la construcción de la equidad de género. Muy posiblemente el discurso de la diversidad puede sentirse más cómodo con el enfoque del feminismo poscolonial, porque se rompe con las conceptualizaciones universalistas de los derechos de las mujeres. Los localismos y la especificidad ganan terreno en el espacio simbólico y territorial de las mujeres; de tal manera que la normativa liberal del feminismo globalizado que se ha divulgado por medio de los Estados nacionales, organismos internacionales y organizaciones no gubernamentales, ha sido cuestionada por su concepción homogeneizadora de la igualdad de género.

Por su parte, autoras descolonizadoras señalan que su tarea no refiere solo al cuestionamiento del conocimiento que se ha producido desde Occidente, sino que su tarea implica también cuestionar el conocimiento que se ha producido desde los mismos movimientos políticos de los que ellas a su vez forman parte, "los cuales han tendido a reproducir las representaciones y exclusiones del colonizador" (Suárez y Hernández, 2011).

A pesar de los avances y cambios que se han generado en el campo educativo, político y laboral, entre otros, hay aspectos que no han cambiado o lo hacen de forma lenta y atenuada. Tal es la desigualdad en el reparto del trabajo doméstico, cuidado y crianza de los hijos y las hijas y personas dependientes, que impiden una distribución equitativa de las responsabilidades familiares y que siguen incidiendo, no como factores únicos, pero sí determinantes, para que las mujeres se desarrollen en otros espacios de forma equitativa e igualitaria. Para ello hace falta que a la existencia de la "tercera mujer", concepto que utiliza Lipovestky (2007) para referirse a las mujeres contemporáneas que rompen con patrones tradicionales, se une la presencia del "tercer hombre" para que secunde las trasformaciones que impulsan las mujeres y otros colectivos a fin de que impacten en las históricas -y desiguales- relaciones de poder. Se requiere romper con patrones propios de la sociedad capitalista y heterosexista para permutarla. Los discursos en pro de la defensa de los derechos de las mujeres y muchas minorías promueven cambios, pero no es suficiente, se necesitan nuevas prácticas, no solo discursos que revolucionen el mundo de lo cotidiano y construyan nuevas estructuras sociales y de pensamiento. A lo mejor, el discurso o los discursos que se generan a partir del poscolonialismo contribuyan a construir las rupturas estructurales que hasta el momento no se ha logrado obtener.

\section{Conclusiones}

Los aspectos teóricos y hechos sociales planteados nos permiten realizar la siguiente reflexión de cierre:

- La década de finales de los años ochenta y el noventa del siglo pasado se caracterizaron por ser un período álgido del desarrollo del movimiento de mujeres en Costa Rica. Durante la primera década 
del presente siglo, se genera un impase paulatino en los movimientos de mujeres y espacios de coordinación nacional dejan de existir o tener la presencia política que tuvieron en las dos décadas anteriores, como es el caso de la Agenda Política de las Mujeres (1997).

- Una vez obtenida cierta presencia y logros en las demandas durante finales del siglo XX y la primera década del XXI, se genera un proceso de cooptación de las dirigentes y, en general, de los movimientos sociales de mujeres que, por razones personales de distinta índole, empezaron a trabajar en instituciones públicas, organismos internacionales, organizaciones no gubernamentales. Igualmente, son mujeres que o retornan o más bien se concentran en el trabajo de sus partidos políticos, sindicales u otros, con el objetivo en algunos casos de fortalecer desde allí demandas propias de las mujeres. De esta manera se generalizó socialmente el enfoque de género en distintas instancias públicas y se generaron propuestas específicas de políticas públicas e insumos para programas nacionales incorporando el enfoque de género. En palabras de Mirta González (2006), el movimiento de mujeres costarricenses de fin de siglo llegó a una maduración consensual en torno a aspectos tales como la equidad por medio de cuotas de representación política, la responsabilidad paterna, así como la necesidad de desarrollar políticas públicas con perspectiva de género. Los movimientos se vieron debilitados paulatinamente por dicha institucionalización y actividades y acciones que antes se organizaban desde este. Todo ello abundó en la generación de un proceso paulatino de institucionalización de la lucha de las mujeres.

- La formación con perspectiva de género que reciben grupos de mujeres y grupos afines a la defensa de los derechos de las mujeres y minorías, que se oponen al sistema heterosexista de nuestra sociedad, no es suficiente, tal y como lo demuestran experiencias que se han dado por medio de programas gubernamentales; tales como los impulsados por el INAMU, el IMAS, u otros, en oposición a la formación que recibieron muchas mujeres en la década de los ochenta y noventa del siglo pasado por grupos feministas como Alianza de Mujeres, El Colectivo de Mujeres Pancha Carrasco y Cefémina, entre otros. La perspectiva política de la lucha de la mujeres es fundamental para avanzar en aspectos políticos, así las demandas estratégicas de género siguen siendo una bandera de lucha de muchas mujeres que se plantean, de forma consciente, romper con cánones, esquemas y estructuras propias de la sociedad actual.

- Los movimientos sociales son forjadores de sujetos político-económicos y culturales, y justamente por ser todo ello no debería ser cooptado por la militancia política partidaria, sindical u otra, o por las instituciones gubernamentales. Su riqueza estriba en la posibilidad de retroalimentar y alimentar esos espacios desde sus demandas particulares, las cuales pierden fortaleza al ser cooptado por cualquiera de los espacios de participación política o institucional. El distanciamiento estratégico es la naturaleza propia de cualquier movimiento para continuar en uno de sus particulares ciclos de desarrollo, sea de lucha álgida o de gestación de nuevos espacios y el fortalecimiento de estos de cara a las nuevas coyunturas que acontecen en el campo social, económico, cultural, sea nacional, global o internacional.

- No cabe duda que los movimientos sociales devienen cada vez más amplios y diversos, que las demandas y los sujetos colectivos e individuales que los forman cada vez son más heterogéneos. Los movimientos de las mujeres muestran esa diversidad que se ha ampliado con los colectivos LGBTI, colectivos e individuos que a pesar de sus intereses particulares se han mostrado sensibles y se identifican con las luchas de las mujeres en general y con las lésbico/feministas en particular, y sus actividades van más allá al vincularse a procesos de luchas generadas desde otros espacios, conformando alianzas muy potentes. 
- Los colectivos LGBTI han ganado mayor protagonismo en los últimos años, algunos devienen de grupos feministas-lésbicos u homosexual, pero en la actualidad sus demandas se han diversificado. Quienes forman parte de los distintos grupos pueden sentirse identificadas(os) con distintas corrientes del feminismo pero a veces se distancian del mismo por ser un movimiento que, hasta hace poco, se había centrado en el estudio y prácticas de lucha que giran en torno a las mujeres como representantes de las desigualdades propias de un sistema capitalista con un claro sesgo heterosexista, fundado en la lógica de la dominación masculina y anclado en prácticas patriarcales.

\section{Referencias}

Barahona, Macarena (1987). La lucha de las mujeres por el voto. San José, UCR, Tesis para optar al grado de licenciatura en sociología.

Bedoya, Mauricio H. (2013). Revista Virtual Universidad Católica del Norte, 40 (162) SeptiembreDiciembre 162-173.

Castells, Manuel. (2001). La galaxia Internet. Aretè: Madrid.

Castells, Manuel. (1998). La era de la información- Economía, sociedad y cultura. Vol. 2. El poder de la identidad, Alianza Editorial, Madrid. 496 p.

Cepal. (2012). La política de paridad y alternancia en la Ley Electoral de Costa Rica. Un avance en la garantía de la autonomía en la toma de decisiones de las mujeres. Observatorio de Igualdad de Género de América Latina y el Caribe, Naciones Unidas. 126 p.

Camacho, Rosa L.; Lara, Silvia y Serrano, Esther (1996). Las cuotas mínimas de participación de las mujeres. Un mecanismo de acción afirmativa (aportes para la discusión). Centro Nacional para el Desarrollo de la Mujer y la Familia (ahora Instituto de la Mujer). San José.

Cordero, Teresita (1996). Organizaciones de mujeres: apertura de espacios no tradicionales por mujeres campesinas. Tesis de Maestría en Psicología. Universidad de Costa Rica, San José. 270 p.

Ciriza, Alejandra (2007). Movimientos sociales y ciudadanía: notas sobre la ambivalencia ante el espejo de lo colectivo. Rev. La Aljaba. Volumen XI: 27-43.

Fallas, Ligia (2014). Entrevista Realizada a la diputada Ligia Fallas, Partido Frente Amplio (14 de mayo 2014).

Flores, Ana Lorena (1997). La fuerza de lo cotidiano en la organización de mujeres campesinas: el caso de las mujeres de MUSA. Tesis de Licenciatura. Universidad de Costa Rica, San José. 300 p.

Flores, Cinthia y Jiménez, Andrea. (2013). El movimiento social de mujeres como plataforma de la participación política: un espacio para la concienciación y empoderamiento. Tesis de licenciatura, Universidad de Costa Rica. 182 p.

Foucault, Michel (1978). "Verdad y poder”. En Microfísica del poder, Madrid, La Piqueta.

González, Mirta (2006). "La agenda política del movimiento de mujeres: Demandas de fin de siglo". Rev. Reflexiones 85 (1-2): 157-170.

Instituto Nacional de las Mujeres (2007). Política Nacional para la Igualdad y Equidad de Género. INAMU, UNFPA. Costa Rica. 97 p.

Lara, Silvia (1994). Feminismo y cambio social: el caso de las mujeres dirigentes en la lucha por vivienda en Costa Rica. San José, UCR, tesis para optar al grado de maestría, Maestría Centroamericana de Sociología. 305 p.

Lipovetsky, Gilles (2007). La tercera mujer. Anagrama, Barcelona. 297 p.

Moreno, Elsa (1995). Mujeres y política en Costa Rica. FLACSO Costa Rica; 1995. 172 p.

Moser, Caroline (1992). Género y desarrollo. FLACSO, San José. 105 p.

Moser, Caroline (1995). Planificación de género y desarrollo: Teoría, práctica y capacitación. Lima, Flora Tristán. 65 p.

Offe, Claus (1985). New social movements: challenging the boudaries of institutional politics. En: Social Rearch 52. 4. 
Piedra, Nancy (2011). Amor/desamor: relaciones de poder en las parejas y vínculos amorosos en la Costa Rica urbana contemporánea. Tesis de doctorado, El Colegio de México, México DF. 400 p.

Piedra, Nancy (1999). "Mujeres rompiendo barreras: promotoras de cambios, protagonistas de luchas; cultura política de las mujeres" En: Revista de Ciencias Sociales 84-85. II, III 1999, Costa Rica.

Piedra, Nancy (1998) Sueños inconclusos y caminos de esperanza: Acerca de la construcción en la identidad de género en mujeres del sector urbano popular, San José, Tesis de la Maestría, Universidad de Costa Rica. 300 p.

Tigat-Pflaum (2014). Tres actos del feminismo: Nancy Fraser y los debates feministas de los últimos 40 años. Rev. Nueva sociedad 251 (5-6): 127-135.

Sagot, Montserrat (1997). The struggle for husing in Costa Rica: the transformation of women into political actors. En: The Costa Rican wommen's movement. (Ilse Abshagen Leitinger, compiladora). University of Pittsburgh Press, Pittsburgh.

Serrano, Esther (2002). De la memoria individual a la historia social: grupos de encuentro de las mujeres lesbianas costarricenses. Tesis de Maestría, Universidad Nacional de Heredia, Costa Rica. 200 p.

Suárez, Liliana y Hernández, Rosalva (eds) (2011). Descolonizando el feminismo, teorías y prácticas desde los márgenes. Ediciones Cátedra, Universidad de Valencia, Instituto de la Mujer, España. 454 p.

Torres, Paulina (2012). Formulación de una propuesta para fortalecer el trabajo de incidencia política de la organización Colectiva por el Derecho a Decidir - CPDD, dirigido a las mujeres lesbianas. Tesis de Licenciatura, Universidad de Costa Rica. 192 p.

Touraine, Allan (1995). Producción de la sociedad. Instituto de Investigaciones Sociales, Universidad Autónoma de México, Instituto Francés de América Latina y Embajada de Francia, México 372 p.

Vargas, Virginia (1992). Como cambiar el mundo sin perdernos: el movimiento de mujeres en el Perú y en América Latina. Ediciones Flora Tristán, Lima, Perú.

Vargas, Virginia (1997). Ciudadanía. Manuscrito no publicado. Lima.

Young, Kate (1993). "Planning from a gender perspective: Making a world of difference" En: Kate Young, Planning Development with Women: Making a World of Difference. Macmillan, London $187 \mathrm{p}$.

\section{Páginas web visitadas}

Anónimo, Red de Mujeres de Costa Rica, visita realiza el 25/06/2014, http://mujeresrurales.org/.

Anónimo (2014). Ley de Sociedades de Convivencia, Expediente N..o 18.481, visita realizada 23/06/2014 (http://www.asamblea.go.cr/Centro_de_informacion/Sala_de_Prensa/Documents/ Exp.18481.pdf).

Anónimo (2014) Agenda de las mujeres, visita realizada 20/06/2014.http://www.agendadelasmujeres. com.ar/paginas/costarica.html. 
